\title{
Comparative study of State Estimation of Fuel Cell Hybrid System Using UKF and EKF
}

\author{
Rambabu Kandepu, Biao Huang, Lars Imsland and Bjarne Foss
}

\begin{abstract}
The principle of state estimation of a dynamic system using Unscented Kalman Filter (UKF) and Extended Kalman Filter (EKF) is briefly described. Two nonlinear examples are considered to compare the state estimation using UKF and EKF. A Solid Oxide Fuel Cell (SOFC) combined with Gas Turbine (GT) hybrid system is described and system level modeling of the hybrid system is discussed. The state estimation using UKF and EKF is applied to the fuel cell hybrid system.
\end{abstract}

\section{INTRODUCTION}

State estimation plays a significant role in control and monitoring. As all the practical systems have some degree of nonlinearity and the nonlinear state estimation has to be taken into consideration for designing an advanced control system and for monitoring purpose. The state estimation is a means to propagate the probability distribution function (pdf) of the state of the system over time and to update the pdf using the measurements from the system. Kalman Filter (KF) is the most commonly used estimator for linear systems. KF is optimal if the system is linear and noises are Gaussian. The Gaussian distribution can be represented by two parameters at a time instant: mean and variance. KF propagates the mean and variance in an optimal (minimum mean square error) way. If the system cannot be approximated by a linear function in its operating region, nonlinear state estimation is necessary for an optimal control design and effective monitoring purpose. The most common way of applying KF for the nonlinear system is in the form of Extended Kalman Filter (EKF). In EKF, the pdf is propagated through a linear approximation of the system around the operating point at that time instant. In doing so, the EKF needs the Jacobian matrices which makes it difficult for higher order systems, for online state estimation. Julier and Uhlmann [3]developed an alternative method for the nonlinear estimation: Unscented Kalman Filter (UKF). The UKF propagates the pdf in a simple and effective way and it is accurate up to second order in estimating mean and covariance [3].

In the foreseeable future, fossil fuels including natural gas will be a major source of energy. With today's increasing concern about global warming and climate change, there is an incentive to investigate natural gas power processes

This work was supported by The Gas Technology Center, NTNUSINTEF, NFR and Western Canada Fuel Cell Initiative (WCFCI).

K. Rambabu and B. Foss are with Department of Engineering Cybernetics, Norwegian University of Science and Technology, Trondheim, Norway

\{Rambabu.Kandepu,Bjarne.Foss\}@itk.ntnu.no

B. Huang is with Chemical and Materials Engineering, University of Alberta, Edmonton, Alberta, Canada Biao. Huang@ualber ta.ca

L. Imsland is with SINTEF, IKT, Trondheim, Norway Lars.Imsland@sintef.no that operate efficiently, thus emitting less per kWh produced, and also investigate power production processes with $\mathrm{CO}_{2}$ capture capabilities. It is widely accepted that fuel cells are power sources that will become increasingly important due to high efficiency, low levels of pollution and noise, and high reliability. One of the most promising fuel cell technologies is the Solid Oxide Fuel Cell (SOFC) due to its solid state design and internal reforming of gaseous fuels, in addition to its high efficiency. The SOFC converts the chemical energy of fuel directly to electrical energy. Since SOFCs operate at high temperatures (about $1000^{\circ} \mathrm{C}$ ), natural gas can be used directly as fuel. The electrical efficiency of a SOFC can reach 55\%. Another significant advantage of the SOFC is that since it operates at high temperature and its efficiency increases when pressurized, it naturally lends itself as a heat source for a gas turbine (GT) cycle. The combined (hybrid) cycle can theoretically have an overall electrical efficiency of up to $70 \%$. with a power range from a few hundred $\mathrm{kWs}$ to a few MWs. Processes based on SOFCs can be used as power sources with $\mathrm{CO}_{2}$ capture, since the "used fuel" (and water) and air exit streams can be kept separated. The main applications of the hybrid system include remote area power supply and distributed power generation.

The paper is organized as follows: Section II and III explain the principle of UKF and EKF respectively. Section IV applies the UKF and the EKF to two engineering applications and compares the performances of UKF and EKF for the state estimation. Section V describes the SOFC/GT hybrid system and state estimation using UKF and EKF. Section VI gives the conclusions and states future work.

\section{STATE ESTIMATION OF NONLINEAR SYSTEMS}

\section{A. UKF}

The principle of the UKF is explained with the following example: let $x$ be a random variable and

$$
y=f(x)
$$

be a nonlinear function. The question is how the UKF approximate the propagation of pdf of $x$ ? For example, in the case of Gaussian distribution, how to calculate the mean $(\bar{y})$ and covariance $\left(\Sigma_{y}\right)$ of $y$ ? Consider a set of sigma points $x^{(i)}$, (similar to the random samples of a specific distribution function in Monte Carlo simulations) with each point being associated with a weight $w^{(i)}$. Both the sigma points and the weights are computed deterministically through a set of conditions given in [3]. Then the following steps are involved in approximating the mean and covariance: 
1) Propagate each sigma point through the nonlinear function,

$$
y^{(i)}=f\left(x^{(i)}\right)
$$

2) Mean is the weighted average of the transformed points,

$$
\bar{y}=\sum_{i=0}^{p} w^{(i)} y^{(i)}
$$

3) The covariance is the weighted outer product of the transformed points,

$$
\Sigma_{y}=\sum_{i=0}^{p} w^{(i)}\left(y^{(i)}-\bar{y}\right)\left(y^{(i)}-\bar{y}\right)^{T}
$$

The UKF algorithm is presented below; for the fundamental theory, refer to [1], [2]. Let the system be represented by the following standard discrete time equations:

$$
\begin{gathered}
x_{k}=f\left(x_{k-1}, v_{k-1}, u_{k-1}\right) \\
y_{k}=h\left(x_{k}, n_{k}, u_{k}\right)
\end{gathered}
$$

where $x$ is the system state, $v$ the process noise, $n$ the observation noise, $u$ the input and $y$ the noisy observation of the system. An augmented state at time instant $k$,

$$
x_{k}^{a}=\left[\begin{array}{l}
x_{k} \\
v_{k} \\
n_{k}
\end{array}\right]
$$

is defined. The augmented state variable dimension is,

$$
L=L_{x}+L_{v}+L_{n}
$$

where $L_{x}$ is the original state dimension, $L_{v}$ is the process noise dimension and $L_{n}$ is the observation noise dimension. Similarly, the augmented state covariance matrix is built from the covariance matrices of $x, v$, and $n$ :

$$
P^{a}=\left[\begin{array}{ccc}
P_{x} & 0 & 0 \\
0 & R_{v} & 0 \\
0 & 0 & R_{n}
\end{array}\right]
$$

where $R_{v}$ and $R_{n}$ are the process and observation noise covariance matrices.

\section{1) Algorithm::}

\section{- Initialization :}

$$
\begin{aligned}
\widehat{x}_{0}=E\left[x_{0}\right], & P_{x_{0}}=E\left[\left(x_{0}-\widehat{x}_{0}\right)\left(x_{0}-\widehat{x}_{0}\right)^{T}\right] \\
\widehat{x}_{0}^{a} & =E\left[x^{a}\right]=E\left[\begin{array}{lll}
\widehat{x}_{0} & 0 & 0
\end{array}\right]^{T} \\
P_{0}^{a} & =E\left[\left(x_{0}^{a}-\widehat{x}_{0}^{a}\right)\left(x_{0}^{a}-\widehat{x}_{0}^{a}\right)^{T}\right] \\
& =\left[\begin{array}{ccc}
P_{x} & 0 & 0 \\
0 & R_{v} & 0 \\
0 & 0 & R_{n}
\end{array}\right]
\end{aligned}
$$

- For $k=1,2, \ldots \infty$ :

1) Calculate sigma-points [1]:

$$
\chi_{k-1}^{a}=\left[\begin{array}{lll}
\widehat{x}_{k-1}^{a} & \widehat{x}_{k-1}^{a}+\gamma \sqrt{P_{k-1}^{a}} & \widehat{x}_{k-1}^{a}-\gamma \sqrt{P_{k-1}^{a}}
\end{array}\right]
$$

where $\gamma$ is a scaling parameter.

2) Time-update equations:

$$
\begin{gathered}
\chi_{k / k-1}^{x}=f\left(\chi_{k-1}^{x}, \chi_{k-1}^{v}, u_{k-1}\right) \\
\widehat{x}_{k}^{-}=\sum_{i=0}^{2 L} w_{i}^{(m)} \chi_{i, k / k-1}^{x} \\
P_{x_{k}}^{-}=\sum_{i=0}^{2 L} w_{i}^{(c)}\left(\chi_{i, k / k-1}^{x}-\widehat{x}_{k}^{-}\right)\left(\chi_{i, k / k-1}^{x}-\widehat{x}_{k}^{-}\right)^{T}
\end{gathered}
$$

3) Measurement-update equations:

$$
\begin{gathered}
y_{k / k-1}=h\left(\chi_{k / k-1}^{x}, \chi_{k-1}^{n}\right) \\
\widehat{y}_{k}^{-}=\sum_{i=0}^{2 L} w_{i}^{(m)} y_{i, k / k-1} \\
P_{\bar{y}_{k}}=\sum_{i=0}^{2 L} w_{i}^{(c)}\left(y_{i, k / k-1}-\widehat{y}_{k}^{-}\right)\left(y_{i, k / k-1}-\widehat{y}_{k}^{-}\right)^{T} \\
P_{x_{k} y_{k}}=\sum_{i=0}^{2 L} w_{i}^{(c)}\left(\chi_{i, k / k-1}^{x}-\widehat{x}_{k}^{-}\right)\left(y_{i, k / k-1}-\widehat{y}_{k}^{-}\right)^{T} \\
K_{k}=P_{x_{k} y_{k}} P_{\bar{y}_{k}}^{-1} \\
\widehat{x}_{k}=\widehat{x}_{k}^{-}+K_{k}\left(y_{k}-\widehat{y}_{k}^{-}\right) \\
P_{x_{k}}=P_{x_{k}}^{-}-K_{k} P_{\bar{y}_{k}} K_{k}^{T}
\end{gathered}
$$

\section{B. $E K F$}

Consider the same example discussed in (1). If $f$ is a linear function and the pdf of $x$ is a Gaussian distribution, then Kalman Filter (KF) is optimal in propagating the pdf. Even if the pdf is not Gaussian, the KF is optimal up to the first two moments in the class of linear estimators [2]. The $\mathrm{KF}$ is extended to the class of nonlinear systems and it is termed as EKF. In case of a nonlinear function $(f(x))$, the nonlinear function is linearized around the current value of $x$, and the KF theory is applied to get the mean and variance of $y$. In other words, the mean $(\bar{y})$ and variance $\left(P_{y}\right)$ of $y$, given the mean $(\bar{x})$ and variance $\left(P_{x}\right)$ of the pdf of $x$ are calculated as follows:

$$
\begin{gathered}
\bar{y}=f(\bar{x}) \\
P_{y}=G_{\bar{x}} P_{x} G_{\bar{x}}^{T}
\end{gathered}
$$

where $g$ is the linear approximation of $f((x)$ around $\bar{x}$.

The EKF algorithm is presented below for a general nonlinear system represented by equations (5) and (6) [1]. 
1) Algorithm:

- Initialization:

$$
\begin{aligned}
\widehat{x}_{0} & =E\left[x_{0}\right] \\
P_{x_{0}} & =E\left[\left(x_{0}-\widehat{x}_{0}\right)\left(x_{0}-\widehat{x}_{0}\right)^{T}\right] \\
R_{v} & =E\left[(v-\bar{v})(v-\bar{v})^{T}\right] \\
R_{n} & =E\left[(n-\bar{n})(n-\bar{n})^{T}\right]
\end{aligned}
$$

- For $k=1,2, . . \infty$ :

1) Prediction step

- Compute the process model Jacobians:

$$
\begin{aligned}
F_{x_{k}} & =\left.\nabla_{x} f\left(x, \bar{v}, u_{k}\right)\right|_{x=\widehat{x}_{k-1}} \\
G_{v} & =\left.\nabla_{v} f\left(\widehat{x}_{k-1}, v, u_{k}\right)\right|_{v=\bar{v}}
\end{aligned}
$$

- Compute predicted state mean and covariance (time update)

$$
\begin{aligned}
\widehat{x}_{k}^{-} & =f\left(\widehat{x}_{k-1}, \bar{v}, u_{k}\right) \\
P_{x_{k}}^{-} & =F_{x_{k}} P_{x_{k}} F_{x_{k}}^{T}+G_{v} R_{v} G_{v}^{T}
\end{aligned}
$$

2) Correction step

- Compute observation model Jacobians:

$$
\begin{aligned}
H_{x_{k}} & =\left.\nabla_{x} h\left(x, \bar{n}, u_{k}\right)\right|_{x=\widehat{x}_{k}^{-}} \\
D_{n} & =\left.\nabla_{n} h\left(\widehat{x}_{k}^{-}, n, u_{k}\right)\right|_{n=\bar{n}}
\end{aligned}
$$

- Update estimates with latest observation (measurement update)

$$
\begin{aligned}
K_{k}= & P_{x_{k}}^{-} H_{x_{k}}^{T} \\
& \times\left(H_{x_{k}} P_{x_{k}}^{-} H_{x_{k}}^{T}+D_{n} R_{n} D_{n}^{T}\right)^{-1} \\
\widehat{x}_{k}= & \widehat{x}_{k}^{-}+K_{k}\left[y_{k}-h\left(\widehat{x}_{k}^{-}, \bar{n}\right)\right] \\
P_{x_{k}}= & \left(I-K_{k} H_{x_{k}}\right) P_{x_{k}}^{-}
\end{aligned}
$$

\section{EXAMPLES}

Two examples are considered to compare the state estimation using UKF and EKF.

\section{A. Continuous Stirred Tank Reactors (CSTR) in Series}

The system consists of two constant volume reactors cooled by a single coolant stream flowing in a cocurrent fashion. An irreversible, exothermic reaction occurs in the two tanks. The effluent stream from the first reactor serves as the feed stream for the second reactor. The process model consists of four nonlinear ordinary differential equations:

$$
\begin{aligned}
\dot{C}_{A 1}= & \frac{q}{V_{1}}\left(C_{A f}-C_{A 1}\right)-k_{0} C_{A 1} \exp \left(-\frac{E}{R T_{1}}\right) \\
\dot{T}_{1}= & \frac{q}{V_{1}}\left(T_{f}-T_{1}\right)+\frac{(-\Delta H) k_{0} C_{A 1}}{\rho C_{p}} \exp \left(-\frac{E}{R T_{1}}(40)\right. \\
& +\frac{\rho_{c} C_{p c}}{\rho C_{p} V_{1}} q_{c}\left[1-\exp \left(-\frac{h A_{1}}{q_{c} \rho_{c} C_{p c}}\right)\right]\left(T_{c f}-T_{1}\right) \\
\dot{C}_{A 2}= & \frac{q}{V_{2}}\left(C_{A 1}-C_{A 2}\right)-k_{0} C_{A 2} \exp \left(-\frac{E}{R T_{2}}\right)
\end{aligned}
$$
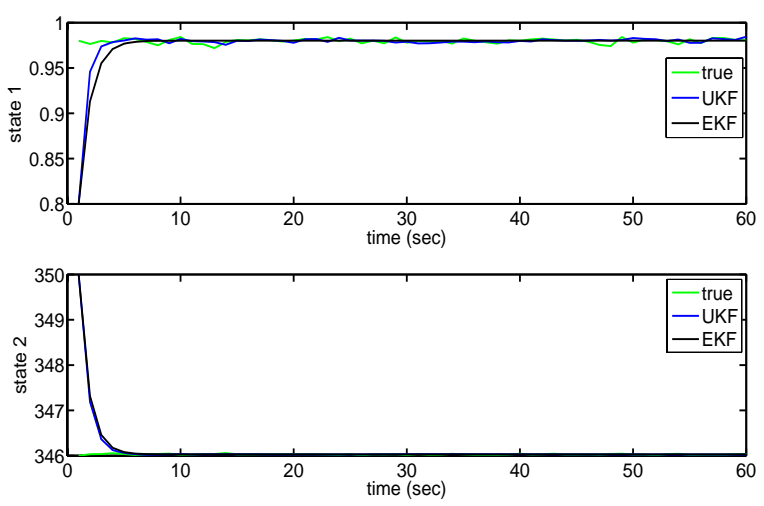

Fig. 1. State estimation of CSTR using UKF and EKF: states 1 and 2

$$
\begin{aligned}
\dot{T}_{2}= & \frac{q}{V_{2}}\left(T_{1}-T_{2}\right)+\frac{(-\Delta H) k_{0} C_{A 2}}{\rho C_{p}} \exp \left(-\frac{E}{R T_{2}}\right) \\
& +\frac{\rho_{c} C_{p c}}{\rho C_{p} V_{2}} q_{c}\left[1-\exp \left(-\frac{h A_{2}}{q_{c} \rho_{c} C_{p c}}\right)\right] \\
& \times\left[T_{1}-T_{2}+\exp \left(-\frac{h A_{1}}{q_{c} \rho_{c} C_{p c}}\right)\left(T_{c f}-T_{1}\right)\right]
\end{aligned}
$$

The same notation as in the [7] is being used, where the subscripts $1,2, c$, and $f$ denote the first reactor, second reactor, coolant stream, and feed stream, respectively. The control objective of the process is to control the effluent composition from the second tank $\left(C_{A 2}\right)$ by manipulating the coolant flow rate $\left(q_{c}\right)$. The state, output and input variables of the process are defined as follows:

$$
\begin{aligned}
x^{T} & =\left[\begin{array}{llll}
C_{A 1} & T_{1} & C_{A 2} & T_{2}
\end{array}\right], \\
y & =C_{A 2}, \\
u & =q_{c}
\end{aligned}
$$

The process is highly nonlinear with the selected input and output variables [7]. The nominal values of all the variables are taken from [7]. The UKF and EKF are used to estimate the states. A white noise with Gaussian distribution is applied on each state and on the output. The simulation results are shown in Figures 1 and 2. The input $\left(q_{c}\right)$ is changed by $50 \%$ at 20th sec.

From the simulation results, it is clear that both the UKF and EKF estimates converge even in the presence of process and observation noises, and a large input disturbance, though the UKF estimate converge faster compared to the EKF. For the EKF, the Jacobian matrices are obtained at each time step by numerical linearization.

\section{B. Continuous Fermentor}

The process consists of a constant volume reactor in which a single, rate limiting substrate promotes biomass growth and product formation. By assuming constant yields, a process model with three differential equations can be obtained [7]. 

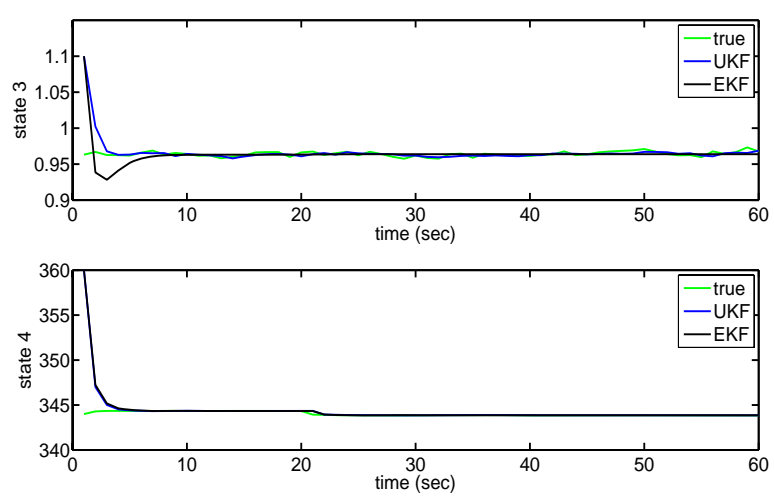

Fig. 2. State estimation of CSTR using UKF and EKF: states 3 and 4

$$
\begin{aligned}
\dot{X} & =-D X+\mu(S, P) X \\
\dot{S} & =D\left(S_{f}-S\right)-\frac{1}{Y_{X / S}} \mu(S, P) X \\
\dot{P} & =-D P+[\alpha \mu(S, P)+\beta] X
\end{aligned}
$$

where $X, S$ and $P$ are the biomass, substrate, and product concentrations, respectively; $D$ is the dilution rate; $S_{f}$ is the feed substrate concentration; and $Y_{X / S}, \alpha$ and $\beta$ are yield parameters. The specific growth rate $\mu$ is modeled as,

$$
\mu(S, P)=\frac{\mu_{m}\left(1-\frac{P}{P_{m}}\right) S}{K_{m}+S+\frac{S^{2}}{K_{i}}}
$$

where: $\mu_{m}$ is the maximum specific growth rate; and $P_{m}$, $K_{m}$ and $K_{i}$ are constant parameters. Nominal operating conditions are taken from [7]. For this process $D$ is the manipulated variable and $X$ is the controlled variable:

$$
\begin{aligned}
x^{T} & =\left[\begin{array}{lll}
X & S & P
\end{array}\right], \\
y & =X, \\
u & =D
\end{aligned}
$$

A white noise with Gaussian distribution is applied on each state and the output in simulation. The state estimation is performed using both the UKF and EKF and the simulation results are as shown in Figure 3. The input $(D)$ is decreased by a $10 \%$ step at 25 th sec. The initial estimate of the state is taken different from the actual state. From the simulation results, in the presence of noise, UKF performs better compared to the EKF.

\section{SOFC/GT HYBRID SYSTEM}

\section{A. System Description}

A schematic diagram of the SOFC/GT hybrid system integrated in an autonomous power system is shown in Figure 1. Fuel is partially steam reformed in a pre-reformer before it enters the SOFC anode. A part of anode flue gas is
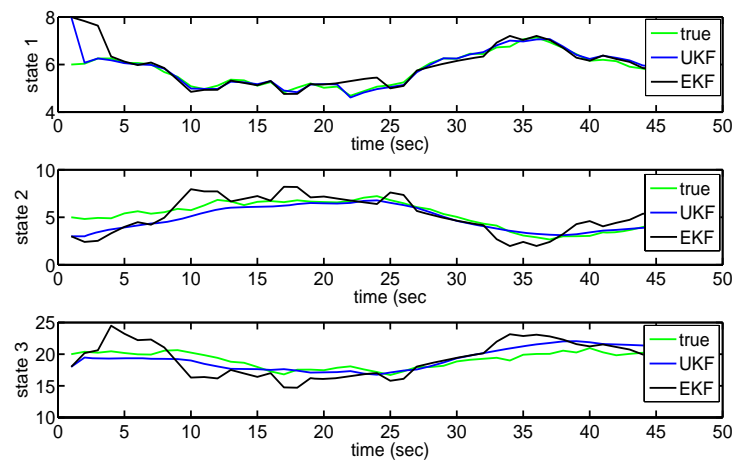

Fig. 3. State estimation of Continuous Fermentor using UKF and EKF: states $1-3$.

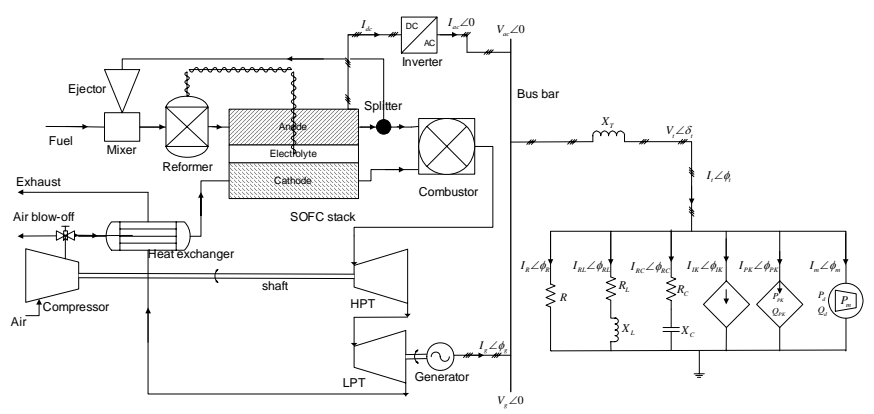

Fig. 4. SOFC-GT hybrid system integrated in an autonomous power system

recycled to supply the necessary steam required for the steam reformation in the pre-reformer. The remaining part of the anode flue gas is supplied to a combustor where the unused fuel is burnt completely in presence of oxygen coming from the cathode flue gas. Air is compressed and preheated in a heat exchanger before entering the SOFC cathode. The hot stream from the combustor is expanded using a High Pressure Turbine (HPT) which drives the compressor. The HPT flue gas is expanded to atmospheric pressure using Low Pressure Turbine (LPT) which drives an alternator. The DC power from SOFC stack is inverted to AC using an inverter. The inverter and the alternator are connected to the electric load through a bus bar. Typically $60-70 \%$ of the total power is supplied by the SOFC stack.

\section{B. Modeling}

All the models of the system are developed in the modular modeling environment gPROMS [6]. The detailed modeling of each component of the system can be found in [4]. A brief description of the SOFC stack model is presented below.

1) SOFC stack: It is assumed that all the SOFCs in the stack operate at identical conditions along the fuel flow direction. A zero-dimensional SOFC model is developed with no regard to the geometry of the cell. The model developed is a lumped one, which includes dynamic molar balances of all the species both in anode and cathode volumes separately. Further, it includes an energy balance treating the whole SOFC as a single volume to model the temperature 


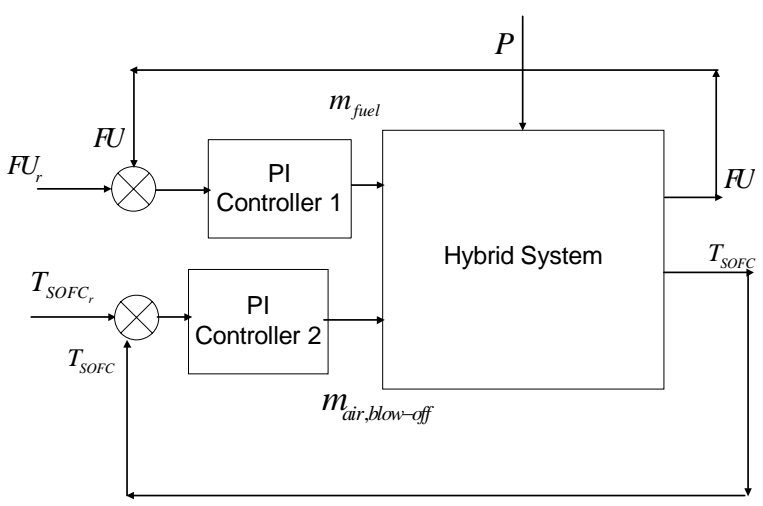

Fig. 5. De-centralized control structure using PI controllers

TABLE I

INPUTS

\begin{tabular}{|l|l|}
\hline No. & Input \\
\hline 1 & FU reference point \\
\hline 2 & SOFC temperature reference point \\
\hline 3 & electric load on the system (measured disturbance) \\
\hline
\end{tabular}

dynamics of the SOFC solid phase mean temperature. There is a radiation from the SOFC to the pre-reformer. The voltage developed across the cell is modeled using Nernst equation, the operating cell voltage is calculated by considering both ohmic and activation losses.

In [4], the low complexity, control relevant SOFC model is evaluated against a detailed model developed in [5]. The comparisons indicate that the low complexity model is sufficient to approximate the important dynamics of the SOFC and can hence be used for operability and control studies.

\section{Regulatory Controller}

Local regulatory control has been considered for the system, and the local control adds additional state to the system. Thus, to design state estimation of the complete system, the local control strategy has to be understood.

A control system is necessary to reject the disturbances such as load changes, fuel and air inlet temperature changes, fuel composition etc. Moreover, during the disturbances, the SOFC temperature should be controlled to prevent cell break down. A decentralized control scheme with two PI controllers is proposed in [4] to reject the disturbances and to control the SOFC temperature. In Figure 5, FU refers to Fuel Utilization, which is defined as the ratio of fuel used in the SOFC and the fuel supplied to the SOFC. It is controlled to the reference value $(0.85)$ by manipulating the fuel flow to the system. The air blow-off flow is manipulated to control the SOFC temperature to the reference value. The controller performs well in terms of tracking [4]; the system efficiency can be improved by optimizing the reference values of the controlled variables. Also there are some constraints which are to be taken into account, for example, steam to carbon ratio at pre-reformer inlet, differential pressure across anode
TABLE II

STATES

\begin{tabular}{|c|c|}
\hline No. & State \\
\hline 1 & Pre-reformer temperature (K) \\
\hline 2 & $\mathrm{H}_{2}$ concentration in pre-reformer (mol) \\
\hline 3 & $\mathrm{CH}_{4}$ concentration in pre-reformer (mol) \\
\hline 4 & $\mathrm{H}_{2} \mathrm{O}$ concentration in pre-reformer (mol) \\
\hline 5 & CO concentration in pre-reformer (mol) \\
\hline 6 & $\mathrm{CO}_{2}$ concentration in pre-reformer (mol) \\
\hline 7 & PI controller 1 integral term \\
\hline 8 & Compressor shaft speed (rad/s) \\
\hline 9 & PI controller 2 integral term \\
\hline 10 & Heat exchanger hot stream temperature (K) \\
\hline 11 & Heat exchanger cold stream temperature (K) \\
\hline 12 & $\mathrm{O}_{2}$ concentration in cathode $(\mathrm{mol})$ \\
\hline 13 & $\mathrm{H}_{2}$ concentration in anode (mol) \\
\hline 14 & $\mathrm{CH}_{4}$ concentration in anode (mol) \\
\hline 15 & $\mathrm{H}_{2} \mathrm{O}$ concentration in anode (mol) \\
\hline 16 & CO concentration in anode (mol) \\
\hline 17 & $\mathrm{CO}_{2}$ concentration in anode (mol) \\
\hline 18 & SOFC outlet temperature (K) \\
\hline
\end{tabular}

TABLE III

OUTPUTS

\begin{tabular}{|l|l|}
\hline No. & Output \\
\hline 1 & Pre-reformer temperature (K) \\
\hline 2 & Shaft speed (rad/s) \\
\hline 3 & Heat exchanger hot stream temperature $(\mathrm{K})$ \\
\hline 4 & Heat exchanger cold stream temperature $(\mathrm{K})$ \\
\hline 5 & SOFC outlet temperature $(\mathrm{K})$ \\
\hline 6 & Combustor outlet temperature $(\mathrm{K})$ \\
\hline 7 & Fuel mass flow rate $(\mathrm{kg} / \mathrm{s})$ \\
\hline 8 & Anode recycle flow rate $(\mathrm{kg} / \mathrm{s})$ \\
\hline 9 & Flow to the combustion chamber $(\mathrm{kg} / \mathrm{s})$ \\
\hline 10 & Air blow-off flow rate $(\mathrm{kg} / \mathrm{s})$ \\
\hline 11 & Air mass flow rate $(\mathrm{kg} / \mathrm{s})$ \\
\hline 12 & SOFC current $(\mathrm{A})$ \\
\hline 13 & SOFC voltage $(\mathrm{V})$ \\
\hline 14 & Generator power $(\mathrm{kW})$ \\
\hline
\end{tabular}

and cathode, compressor surge etc. To achieve this, Model Predictive Control (MPC) is necessary and as a basis to develop the MPC as well as monitoring of the hybrid system, the state estimator is to be designed.

\section{State Estimation}

The SOFC/GT hybrid system is modeled in gPROMS [6] modeling environment. It has 3 inputs, 18 states, and 14 measured outputs which are listed in Tables I, II, and III respectively. The hybrid system model in gPROMS is exported to matlab and is used as function in matlab using the gO:MATLAB package [6]. The state estimator of the hybrid system is designed using both the UKF and EKF and the simulation results are presented in Figures 6 and 7. The initial state estimate is different from the actual initial state and from the simulation results it can be concluded that UKF estimate converges to the true states very fast compared to the EKF estimate.

\section{E. Discussion}

Some experience is needed in tuning the UKF estimator. The initial covariance matrix should be small in size as it determines the distribution of sigma points around the 

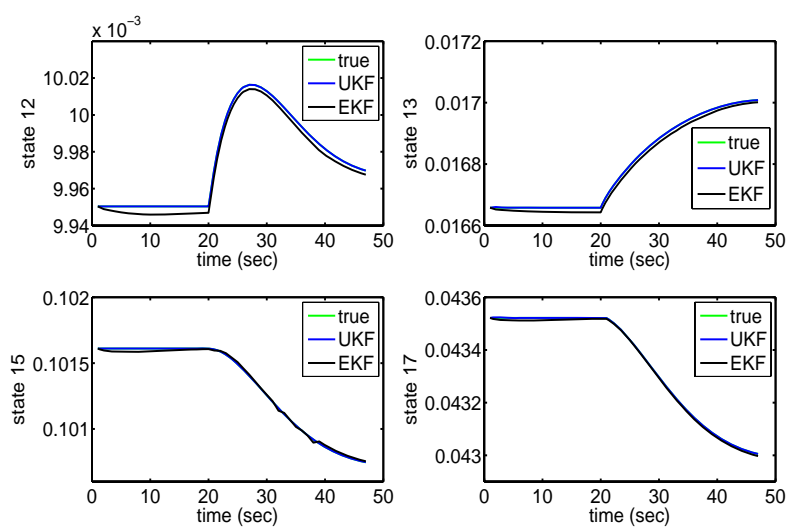

Fig. 6. Comparison of state estimation using UKF and EKF: concentration of $\mathrm{O} 2$ in cathode and concentrations of $\mathrm{H} 2, \mathrm{H} 2 \mathrm{O}$ and $\mathrm{CO} 2$ in anode of SOFC
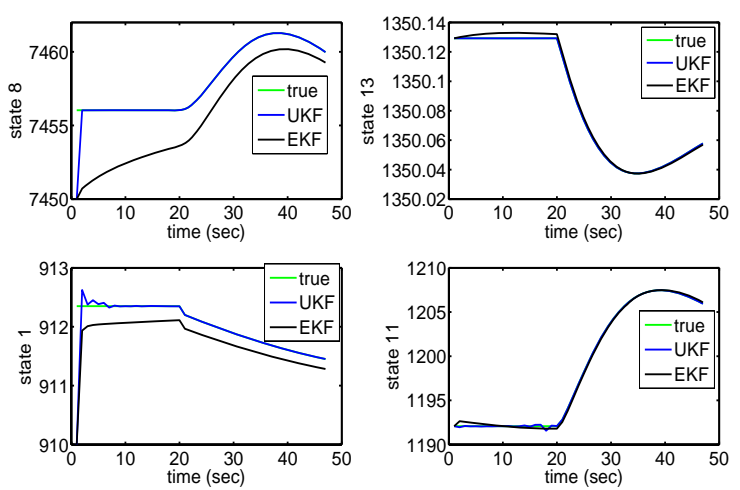

Fig. 7. Comparison of state estimation using UKF and EKF: Compressor shaft speed and SOFC, prereformer and heat exchanger cold stream temperatures

mean. The state covariance matrix should be positive definite at each iteration and the algorithm doesn't guarantee the positive definiteness of the state covariance matrix. Hence the tuning of the UKF estimator is not straight forward. Further the generated sigma points at each iteration should be feasible for the nonlinear transformation; hence sigma point conditioning may be needed depending on the system.

The EKF needs the Jacobian matrices at each iteration and numerical linearization is performed at each step. The size of the perturbation for the numerical linearization is a tuning factor in addition to the state, process noise and measurement noise covariance matrices. The EKF should be properly tuned otherwise it may result in an unstable state estimator.

Overall, the UKF performs better to EKF in terms of the faster convergence in the considered system. If there is no need of numerical linearization, EKF is faster to UKF in terms of computational load, which is not the case in general for the higher order systems. A fair comparison is done between the two estimators as both the estimators are properly tuned to their best.

\section{CONCLUSIONS AND FUTURE WORKS}

\section{A. Conclusions}

The nonlinear estimation using UKF and EKF is performed on two engineering application examples. The results show that the UKF performs better compared to EKF in terms of faster convergence otherwise both the estimators give satisfactory results. The state estimation of SOFC/GT hybrid system is performed using UKF and EKF and the simulation results show that UKF outperforms EKF.

\section{B. Future works}

Future work will be focused on the tuning and positive definite problems mentioned with UKF. The developed estimator will be used to designing a nonlinear MPC.

\section{ACKNOWLEDGMENTS}

Financial support from The Gas Technology Center, NTNU-SINTEF and NFR is acknowledged. We also acknowledge the funding support from Western Canada Fuel Cell Initiative (WCFCI).

\section{REFERENCES}

[1] R. Van der Merwe, "Sigma-Point Kalman Filters for probability inference in dynamic state-space models" PhD thesis, 2004, Oregon Health and Science University.

[2] B. Huang and Q. Wang, "Overview of emerging Bayesian approach to nonlinear system identification". Round tables on Non-linear Model Identification, International Workshop on Solving Industrial Control and Optimization Problems, Cramado, Brazil, April 6-7, 2006.

[3] S. Julier and J. K. Uhlmann, "Unscented filtering and nonlinear estimation", Proceedings of the IEEE, vol. 92, 2004, pp.401-422.

[4] R. Kandepu, L. Imsland, B. Foss, C. Stiller, B. Thorud and O. Bolland, "Modeling and control of a SOFC-GT based autonomous power system", Journal of Energy, 2006, In press.

[5] C. Stiller, B. Thorud, S. Seljbeo, H. Karoliussen and O. Bolland "Finitevolume modeling and hybrid-cycle performance of planar and tubular Solid Oxide Fuel Cells", Journal of power sources, vol. 141, 2005, pp. 227-240.

[6] gPROMS, "gPROMS introductory user guide", Process Systems Enterprise Ltd, 2004.

[7] M. H. Henson, D. E. Seborg, "Nonlinear Process Control", Prentice Hall, NJ, Chapter 4, pp. 198-206, 1997. 